\title{
ИССЛЕДОВАНИЕ ВЕРОЯТНОСТИ БИТОВОЙ ОШИБКИ В РАДИОКАНАЛЕ РЕЛЕЯ В РЕЖИМЕ SІSО ПРИ ИСПОЛЬЗОВАНИИ СВЁРТОЧНЫХ КОРРЕКТИРУЮЩИХ КОДОВ
}

\section{INVESTIGATION OF THE PROBABILITY OF BIT ERROR IN THE RAYLEIGH RADIO CHANNEL IN SSO MODE WHEN USING CONVOLUTIONAL CORRECTION CODES}

M. Seksembayeva N. Tashatov G. Ovechkin

Summary. The article deals with convolutional codes with a given end bit combination (TBC) and Viterbi decoding in a Rayleigh channel with adaptive Gaussian noise. Simulation modeling of the information transmission system using convolutional correction codes and their decoding using the Viterbi method is demonstrated. A linear equalizer with RLS algorithms and a Gain signal amplifier were also used to improve the system's noise immunity. The model was developed using the Matlab software package using the Simulink package. The main indicator of performance of any communication system - the error probabilityis investigated and calculated, and graphs of the error probability dependences on the signal-to-noise ratio (SNR) are constructed. The results obtained can be applied to the selection of an effective type of modulation and a method of noise-tolerant coding in communication systems.

Keywords: rayleigh channel, bit error probability, convolutional codes, Viterbi decoding, intersymbol interference.

\author{
Сексембаева Манара Ануаровна \\ Евразийский национальный университет имени \\ Л.Н. Гумилева \\ manara-1988@mail.ru \\ Ташатов Нурлан Наркенович \\ К.ф.-м.н., дочент, Евразийского начионального \\ университета имени Л.Н. Гумилева \\ tash.nur@mail.ru \\ Овечкин Геннадий Владимирович \\ Д.т.н., профессор, Рязанский государственный \\ радиотехнический университет \\ g_ovechkin@mail.ru
}

Аннотация. В статье рассмотрены сверточные коды с задаваемой концевой комбинацией битов (ТВСС) и декодирование Витерби в канале Релея с адаптивным гауссовским шумом. Продеманстрировано имитационное моделирование системы передачи информации с использованием свёрточных корректирующих кодов и их декодирование по методу Витерби. Для повышения помехоустойчивости системы также были использованы линейный эквалайзер c RLS алгоритмом и усилитель сигнала Gain. Модель разработана при помощи программного комплекса Мatlab с применением пакета Simulink. Исследован и вычислен главный показатель производительности любой системы связи - вероятность ошибки и построены графики зависимостей вероятности ошибки от отношения сигнал/шум (ОСШ). Полученные результаты могут быть применены при выборе эффективного вида модуляции и метода помехоустойчивого кодирования в системах связи.

Ключевые слова: канал Релея, вероятность битовой ошибки, сверточные коды, декодирование Витерби, межсимвольной интерференция.

сверточное кодирование и декодирование Витерби, данные методы исправления ошибок, применяющиеся во многих современных коммуникационных стандартах, таких как LTE и WiMAX и широко используемые в системах связи для улучшения показателей коэффициента ошибок по битам (BER). Таким образом, требуется исследовать вероятность битовой ошибки в многолучевом канале Релея при заданных типах М-арной цифровой модуляции в среде Matlab-Simulink.

Структуры цифровых сигналов, расчет их характеристик, условий их распространения - первичные параметры, которые должны учитываться при проектировании радиосетей. Именно городские условия являются наиболее сложными для распространения радиосигналов. 
Таблица 1. Прохождение сигнала через разные каналы

\begin{tabular}{l|l|} 
Расположение антенн & $\begin{array}{l}\text { Описание } \\
\text { Отражитивный гауссовский канал: Прямая видимость между антеннами, без }\end{array}$ \\
\hline वайсовский канала: Прямая видимость между антеннами, имеется отраженные \\
сигналы.
\end{tabular}

В этих условиях неизбежно многолучевое распространение - отражение от препятствий, дифракция, проникновение сквозь здания и сооружения. Сигнал, приходящий на приемную антенну, имеет задержки и изменение амплитуды во времени. Эти факторы являются наиболее существенными в ухудшении производительности и качества канала. При анализе многолучевых каналов выделяют два их вида — канал Райса и канал Релея.

В каналах Релея и Райса помимо аддитивной составляющей шума присутствуют мультипликативные шумы, вызванные переотражениями и движениями объектов в среде. Прохождение сигнала через канал можно представить следующим образом (Таблица 1):

Один из показателей характеристик качества физического канала это вероятность ошибки (битовой или символьной) при передаче цифрового потока.

Вероятность принятия детектором неверного решения называется вероятностью символьной ошибки, $\mathrm{P}_{E}$.

Битовая ошибка дает количество битов в ошибке в единицу времени. В цифровой связи число битов ошибки - это число битов, принятых в потоке данных по каналу связи, которые изменяются либо из-за шума, помех, искажений, либо из-за битовой синхронизации, затухания, беспроводного многолучевого замирания и т.д.

Соотношение между вероятностью битовой ошибки $\left(\mathrm{P}_{B}\right)$ и вероятностью символьной ошибки $\left(\mathrm{P}_{E}\right)$ для ортогональных М-арных сигналов дается следующим выражением (1):

$$
\frac{P_{B}}{P_{E}}=\frac{2^{k-1}}{2^{k}-1}=\frac{M / 2}{M-1}
$$

Будем рассматривать битовые ошибки. Если коэффициент битовых ошибок в точке приема резко вырастет, то это приведет к неустойчивой связи. Избежав повышения уровня коэффициента битовых ошибок, можно добиться устойчивой связи в точке приема

Основной параметр, определяющий вероятность ошибки в канале - это отношение сигнал/шум. В понятие шум входят и помехи канала. Значения отношения сигнал/шум и вероятности ошибки - обратно пропорциональны. Ниже приведены характеристики вероятности ошибок в канале Релея в сравнении с ошибками в канале Гаусса при одинаковых модуляциях (рис. 1, 2) [1].

Графики показывают огромную разницу между ошибками каналов Релея и Гаусса.

Для исследования уменьшение этой разницы в данной работе был рассмотрен сверточное кодирование с задаваемой концевой комбинацией битов и декодирование Витерби в канале Релея в режиме SISO с адаптивным гауссовским шумом с использованием модуляции BPSK, QPSK и QAM, а их производительность оценивается путем нахождения коэффициента битовых ошибок. Модель создана с помощью Simulink/Matlab 20.

В сверточном коде с задаваемой концевой комбинацией битов начальное и конечное состоянию его матрицы кодирования после кодирования всегда равны. 


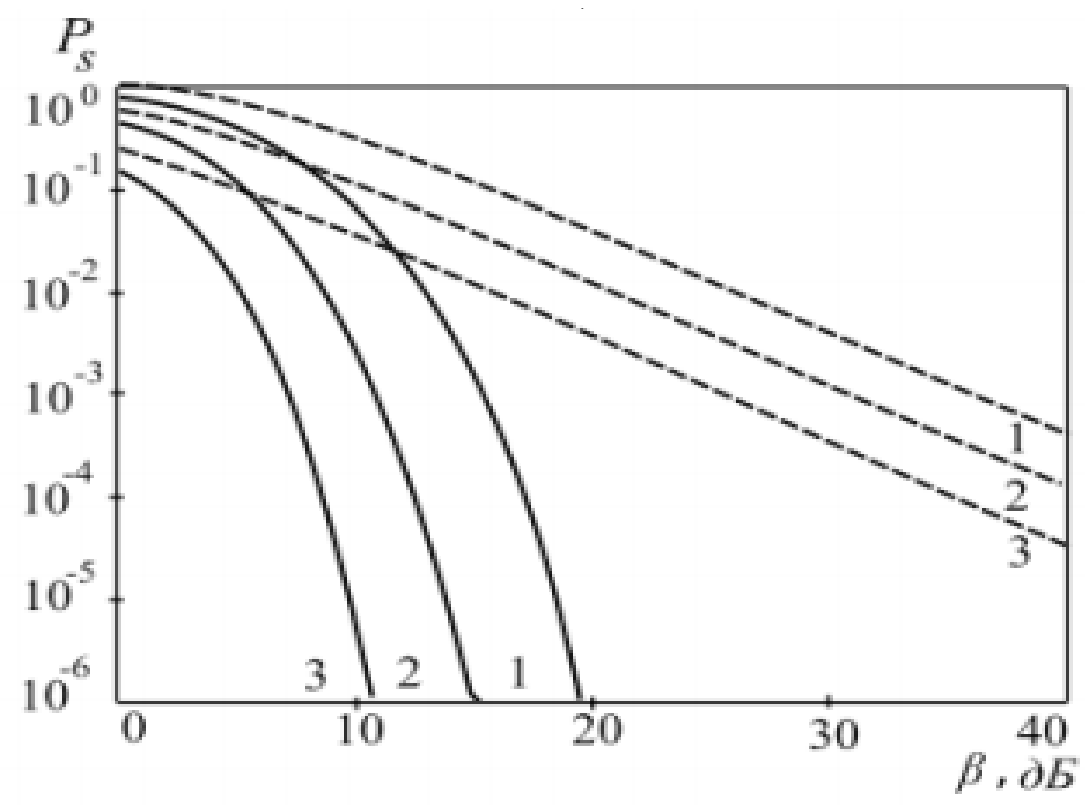

Рис. 1. Вероятности ошибок в канале Релея и Гаусса для модуляции MQAM при: 1) $M=4$;2) $M=16$; 3) $M=64$

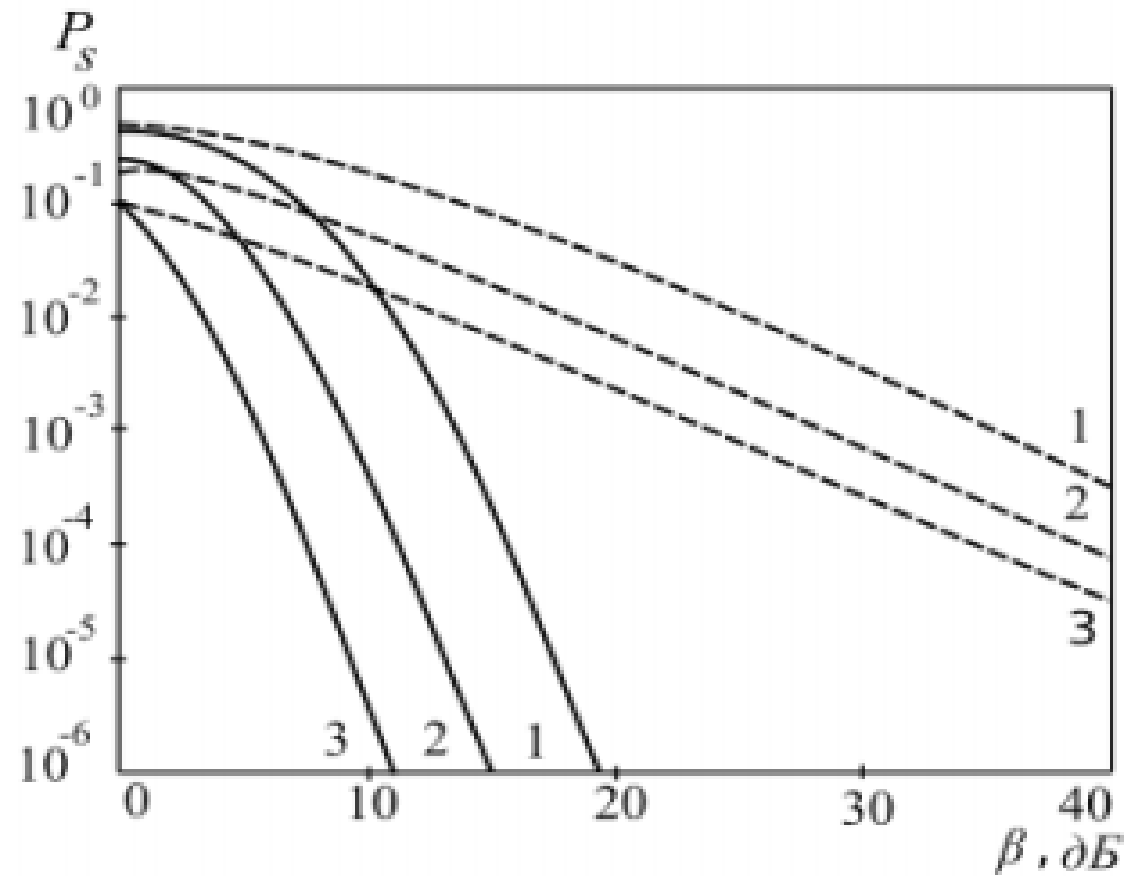

Рис. 2. Вероятности ошибок в канале Релея и Гаусса для модуляции MPSK при: 1) $M=2$;2) $M=8$;3) $M=16$ 


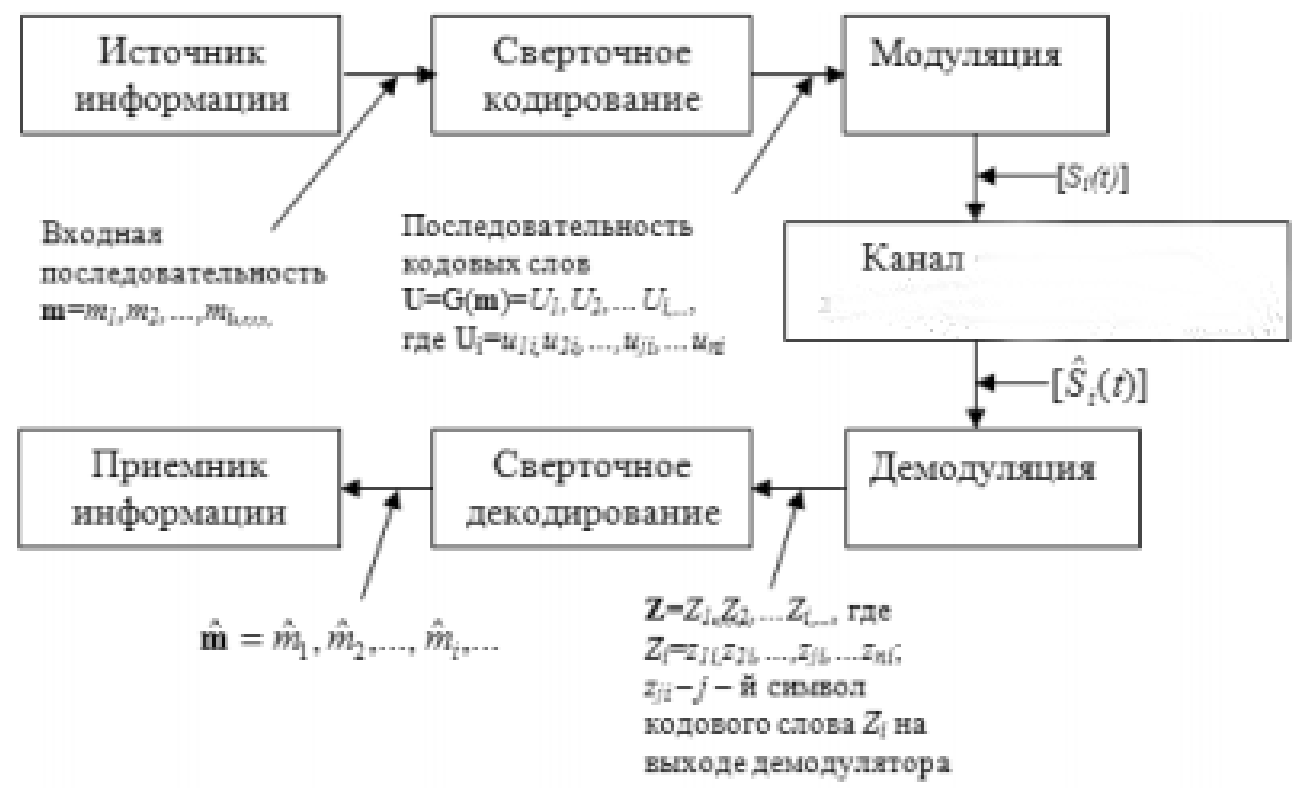

Рис. 3. Кодирование и модуляция в канале связи [2]

Сверточные коды с задаваемой концевой комбинацией битов (ТВСС) - это простые и действенные коды с прямым исправлением ошибок.

Основное практическое различие между сверточным кодом и ТВСС заключается в ограничении завершения. Обычное кодирование сверточным кодом добавляет нулевые биты для наложения нулевых состояний; кодирование ТВСС не требует дополнительных битов, избегая потери скорости

Сверточные коды с задаваемой концевой комбинацией битов (ТВСС) применяют в последнее время многие современные коммуникационные стандарты, такие как LTE $и$ WiMAX.

На следующем рисунке представлена упрощенная функциональная схема сверточного кодирования и модуляции системы передачи информации (рис. 3):

В 1967 г. был разработан алгоритм декодирования Витерби, цель которого уменьшение объема вычислений по сравнению с алгоритмом последовательного декодирования. Преимущество алгоритма Витерби в том, что он не учитывает пути, которые, согласно принципу максимального правдоподобия, не могут быть оптимальными.

Анализ характеристик системы кодирования проводится для наиболее распространенном на практике режима без разнесения антенн. Это режим SISO (Simpleinput-Simpleoutput), т.е. это система с одной передающей и одной приемной антеннами.
Режим SISO основан на реализации двух функциональных блоков: канала с рэлеевскими замираниями и блока адаптивного белого шума. Функциональный блок рэлеевского канала с замираниями представляет в модели эффекты многолучевого распространения в беспроводной среде и влияние на сигнал. Функциональный блок AWGN в модели представляет наложение белого гауссовского шума. Блок AWGN генерирует псевдослучайный шумовой сигнал, характеризуемый гауссовым распределением в частотно-временной области, и последующего сложения уровней сигнала и шума. Реализация функциональной схемы системы передачи информации в Simulink с использованием цифровых схем модуляции BPSK, QPSK и QAM. (Рис. 4)

Для повышения помехоустойчивости системы передачи информации с использованием сверточных алгоритмов обработки сигналов в работе используется линейный эквалайзер с RLS алгоритмом (алгоритм рекурсивных наименьших квадратов) и усилитель сигнала Gain.

Эквалайзер - это устройство, который уменьшает межсимвольную интерференцию.

Адаптивные алгоритмы, алгоритмы рекурсивных наименьших квадратов (RLS) представляет увеличенную сложность, вычислительную стоимость и точность. По сравнению с LMS (Least Mean Square, алгоритм наименьшего квадрата) подход RLS предлагает более быструю сходимость и меньшую ошибку относительно неизвестной системы, за счет требования большего количества вычислений. 


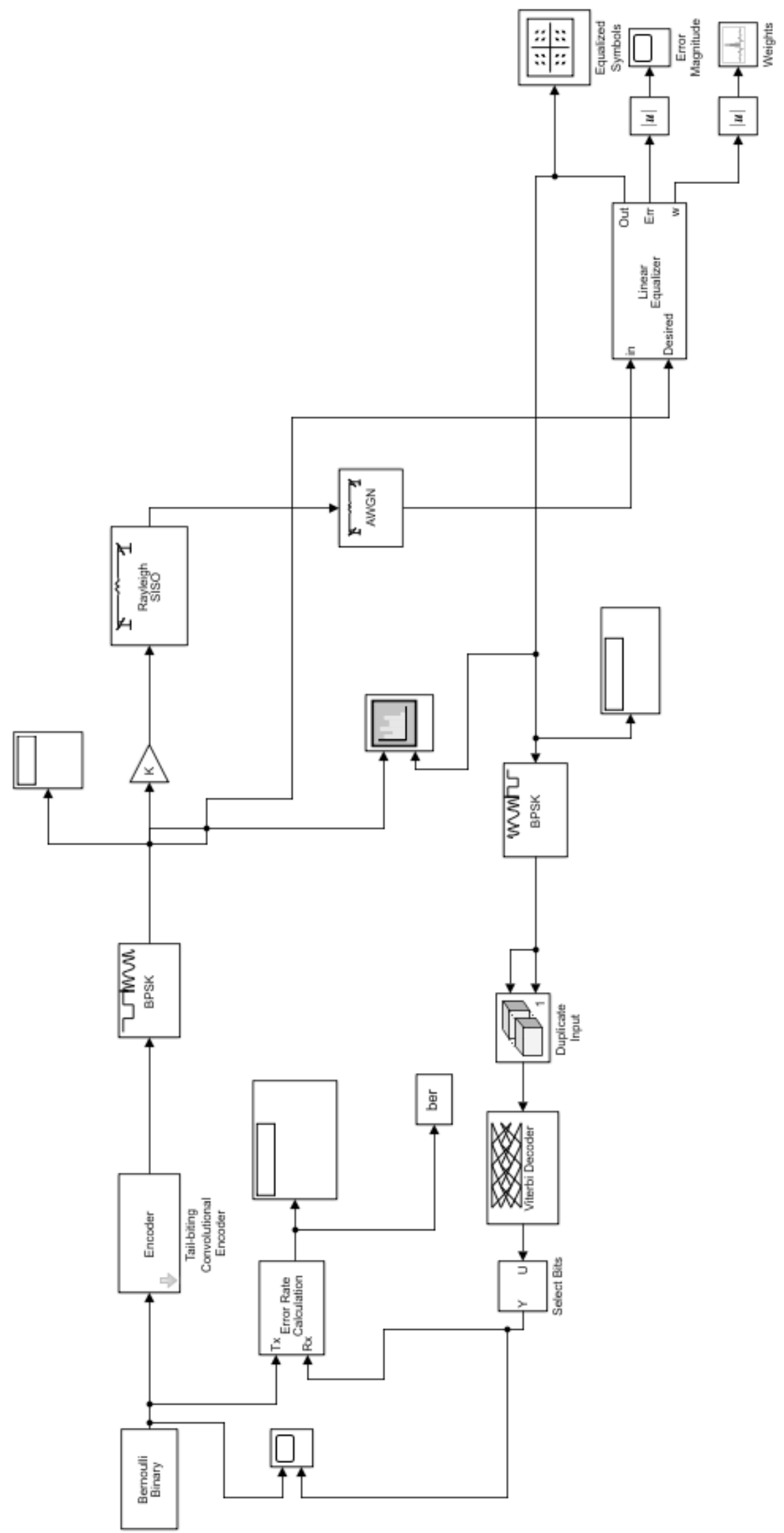

Рис. 4. Блок-схема Simulink с использованием сверточного кодера (ТВСС) и Витерби декодера 


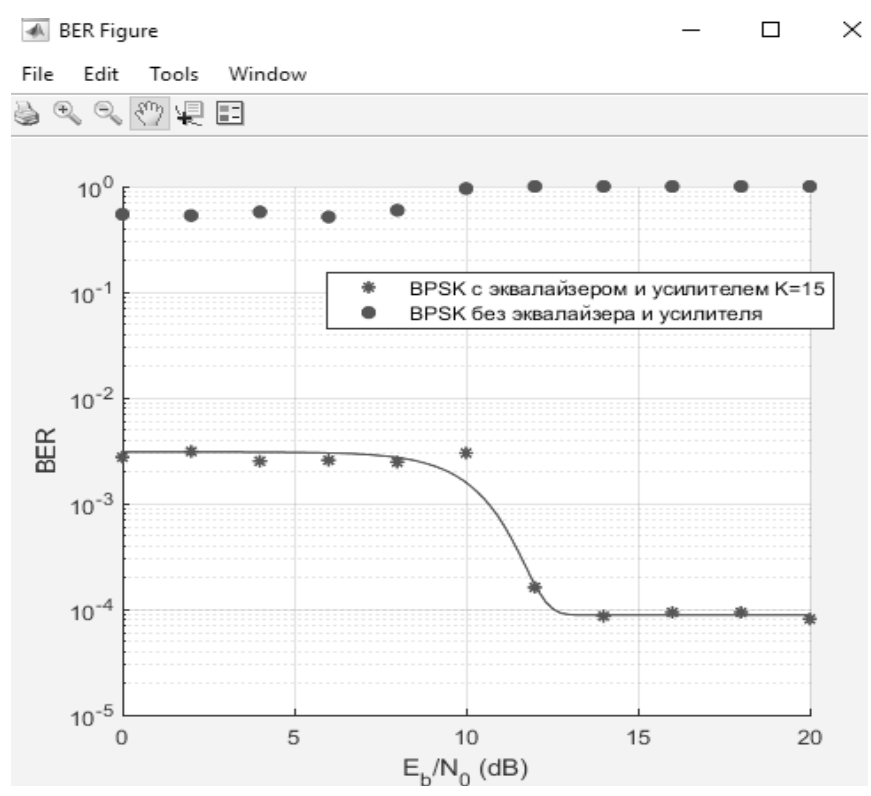

Рис. 5. Вероятности ошибок в канале для модуляции BPSK

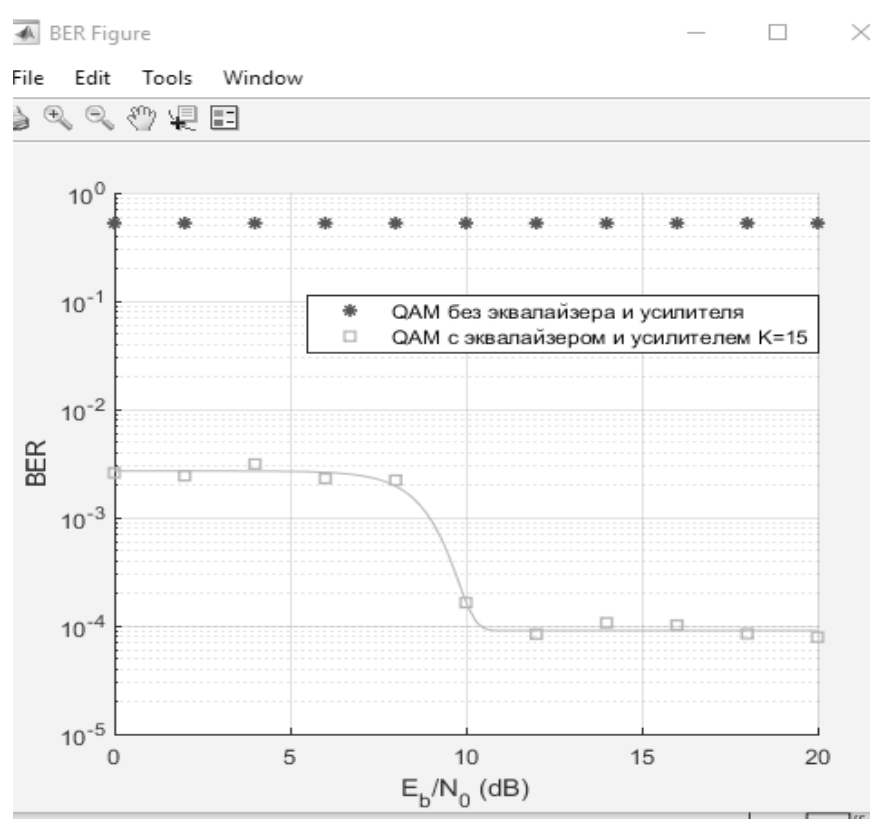

Рис. 7. Вероятности ошибок в канале для модуляции QAM

Линейный усилитель Gain усиливает или ослабляет сигнал в соответвствии с заданным коэффициентом $K[3]$.

$$
S_{b b x}(t)=K^{*} S_{b x}(t)
$$

На рисунках 5-7 можно увидеть, что вероятность ошибки не уменьшается без усилителя и эквалайзера,

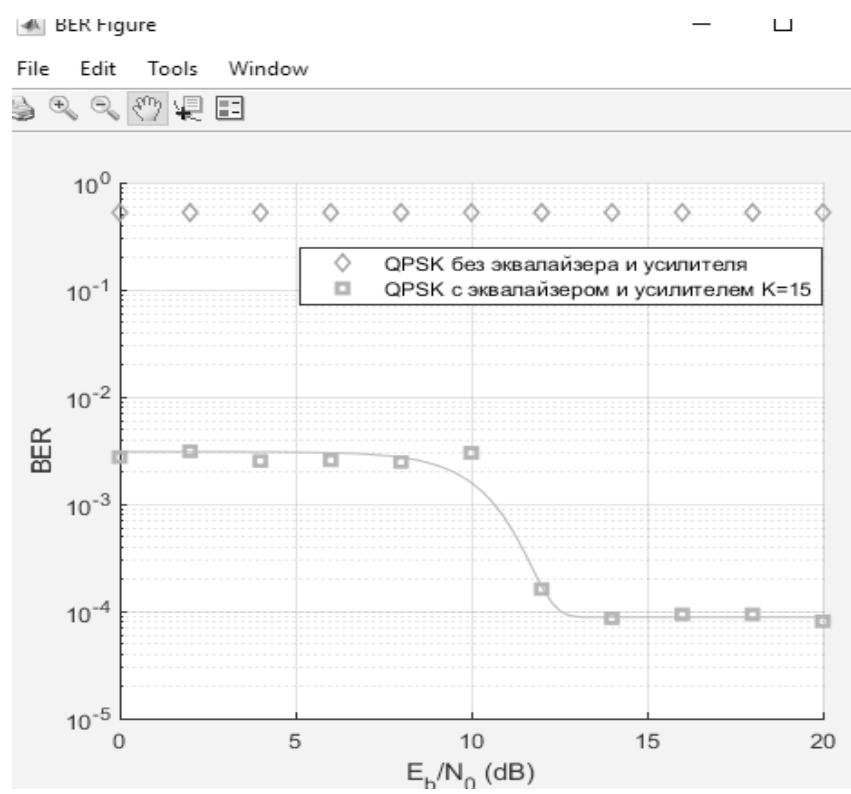

Рис. 6. Вероятности ошибок в канале для модуляции QPSK

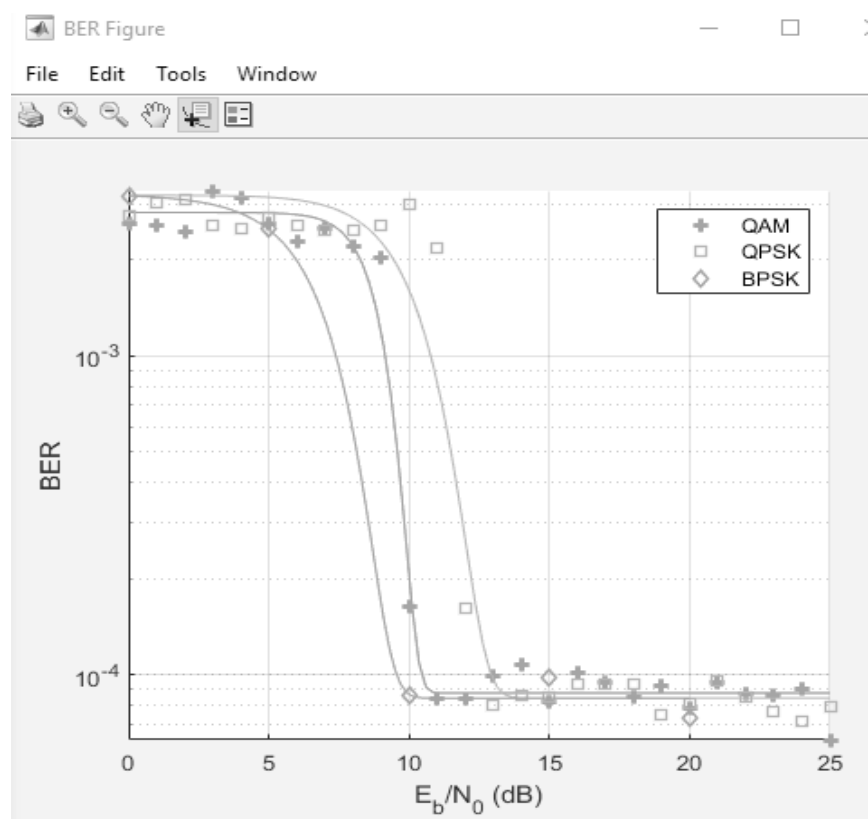

Рис. 8. Характеристика вероятности ошибок в канале Релея при $\mathrm{K}=15$

а с помощью эквалайзера RLS и усилителя Gain можно добиться значительного уменьшения показателей коэффициента битовых ошибок для разных модуляций, при К=15.

А на рис. 8 и 9 объединены сравнительные характеристики вероятности ошибок в канале Релея при разных модуляциях, при коэффициенте усилителя равной $\mathrm{K}=15$ и $\mathrm{K}=30$, соответсвенно 
Таблица 2. Значения битовых ошибок от уровня сигнал/шум при разных типах модуляций

\begin{tabular}{|c|c|c|c|c|c|c|c|c|c|c|c|c|}
\hline \multirow[b]{2}{*}{ 킁 } & \multicolumn{4}{|l|}{ BPSK } & \multicolumn{4}{|l|}{ QPSK } & \multicolumn{4}{|l|}{ 16-QAM } \\
\hline & 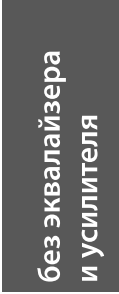 & 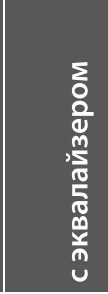 & 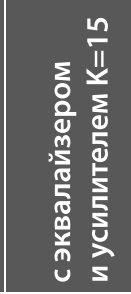 & 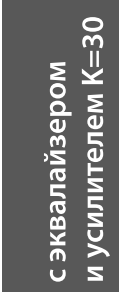 & 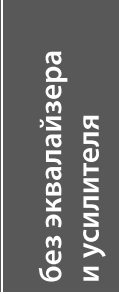 & 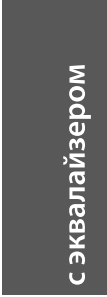 & 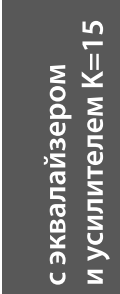 & 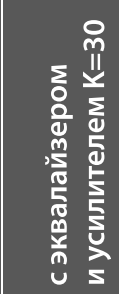 & 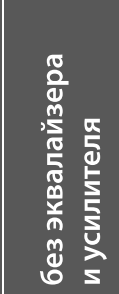 & 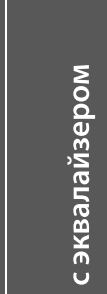 & 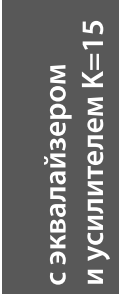 & 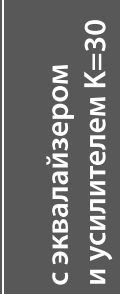 \\
\hline 0 & 0.545 & 0.525 & 0031 & 0.0021 & \multirow{11}{*}{0.525} & 0.49 & 0.0027 & 0.0029 & \multirow{11}{*}{0.525} & 0.5133 & 0.0025 & 0.0022 \\
\hline 2 & 0.53 & 0.4533 & 0.0027 & $8.82 \mathrm{E}-5$ & & 0.4833 & 0.0031 & 0.0024 & & 0.55 & 0.0024 & 0.0022 \\
\hline 4 & 0.575 & 0.5033 & 0.0024 & $8.30 \mathrm{E}-5$ & & 0.535 & 0.0025 & 0.0030 & & 0.505 & 0.0031 & $1.56 \mathrm{E}-4$ \\
\hline 6 & 0.515 & 0.4933 & 0.0022 & $9.04 \mathrm{E}-5$ & & 0.6 & 0.0025 & $1.61 \mathrm{E}-4$ & & 0.51 & 0.0022 & $8.37 \mathrm{E}-5$ \\
\hline 8 & 0.595 & 0.55 & 8.82E-5 & $9.04 \mathrm{E}-5$ & & 0.525 & 0.0024 & $8.60 \mathrm{E}-5$ & & 0.4066 & 0.0022 & $1.05 \mathrm{E}-4$ \\
\hline 10 & 0.955 & 0.0362 & $8.59 \mathrm{E}-5$ & $8.66 \mathrm{E}-5$ & & 0.58 & 0.0030 & $9.33 \mathrm{E}-5$ & & 0.1666 & $1.63 \mathrm{E}-4$ & $1.01 \mathrm{E}-4$ \\
\hline 12 & 1.0 & 0.0055 & 9.04E-5 & $1.02 \mathrm{E}-4$ & & 0.4966 & $1.61 \mathrm{E}-4$ & $9.33 \mathrm{E}-5$ & & 0.0495 & 8.37E-5 & $8.15 E-5$ \\
\hline 14 & 1.0 & 0.0040 & 9.04E-5 & $7.28 \mathrm{E}-5$ & & 0.0641 & $8.60 \mathrm{E}-5$ & 8.07E-5 & & 0.0102 & $1.06 \mathrm{E}-4$ & $8.37 \mathrm{E}-5$ \\
\hline 16 & 1.0 & 0.0039 & $8.66 \mathrm{E}-5$ & $6.17 \mathrm{E}-5$ & & 0.0075 & $9.33 \mathrm{E}-5$ & $7.92 \mathrm{E}-5$ & & 0.0042 & $1.01 \mathrm{E}-4$ & $8.66 \mathrm{E}-5$ \\
\hline 18 & 1.0 & 0.0031 & 8.00E-5 & $7.84 \mathrm{E}-5$ & & 0.0048 & $9.33 \mathrm{E}-5$ & $6.95 \mathrm{E}-5$ & & 0.0036 & $8.44 \mathrm{E}-5$ & $7.63 \mathrm{E}-5$ \\
\hline 20 & 1.0 & 0.0028 & $7.28 \mathrm{E}-5$ & $6.17 \mathrm{E}-5$ & & 0.0038 & $8.07 \mathrm{E}-5$ & $6.56 \mathrm{E}-5$ & & 0.0032 & $7.85 \mathrm{E}-5$ & $6.34 \mathrm{E}-5$ \\
\hline
\end{tabular}

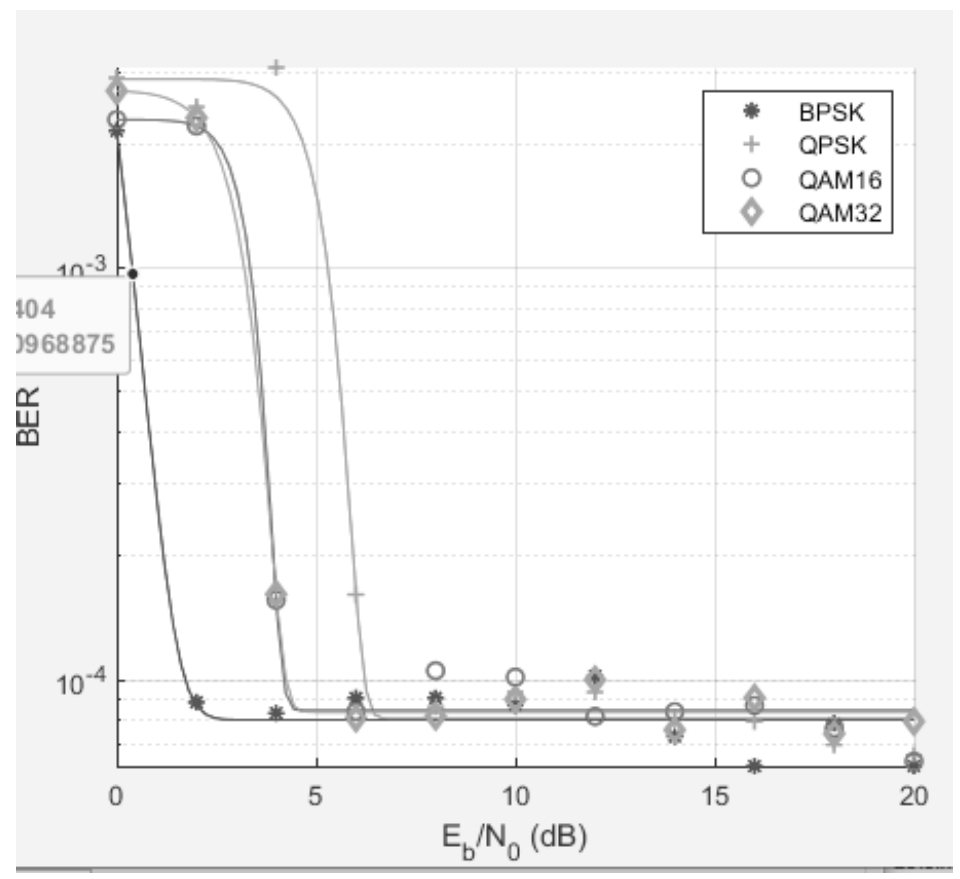

Рис. 9. Характеристика вероятности ошибок в канале Релея при $\mathrm{K}=30$

\section{Bыво}

Если коэффициент битовых ошибок в точке приема резко вырастет, то это приведет к неустойчивой связи. Очевидно, что BPSK имеет более низкий коэффициент битовых ошибок, чем QPSK и M-QAM. Коэффициент би- товых ошибок для тончайшего музыкального сигнала считается равным $10^{-3.5}$ при использовании линейного эквалайзера c RLS алгоритмом и усилителя сигналов, он приблизительно достигается при Eb/No = 7 дБ при коэффициенте 15 и Eb/No = 0,0004 дБ при коэффициенте 30 (Таблица 2). 


\section{ЛИТЕРАТУРА}

1. Ошкина А.В., Петров В.П., «Анализ производительности и качества радиоканалов Релея в режиме SISO», Труды XIV международной научнотехнической конференции актуальные проблемы электронного приборостроения АПЭП — 2018, Том 4 Новосибирск 2018, С. 113-118

2. Сицин Д.В., Повышение помехоустойчивости радиотехнических систем передачи информации с использованием сверточных алгоритмов обработки сигналов: дис. канд. тех. наук. Владимир. гос. Университет. Владимир, 2014

3. Павлейно М.А, Ромаданов В. М., Сафронова Ю. Ф., Статуя А. А., Моделирование работы электрических схем в Simulink. Применение операционных усилителей для фильтрации, усиления и генерации сигналов. УМП — СПб., 20073

(c) Сексембаева Манара Ануаровна ( manara-1988@mail.ru),

Ташатов Нурлан Наркенович ( tash.nur@mail.ru ), Овечкин Геннадий Владимирович ( g_ovechkin@mail.ru ).

Журнал «Современная наука: актуальные проблемы теории и практики»

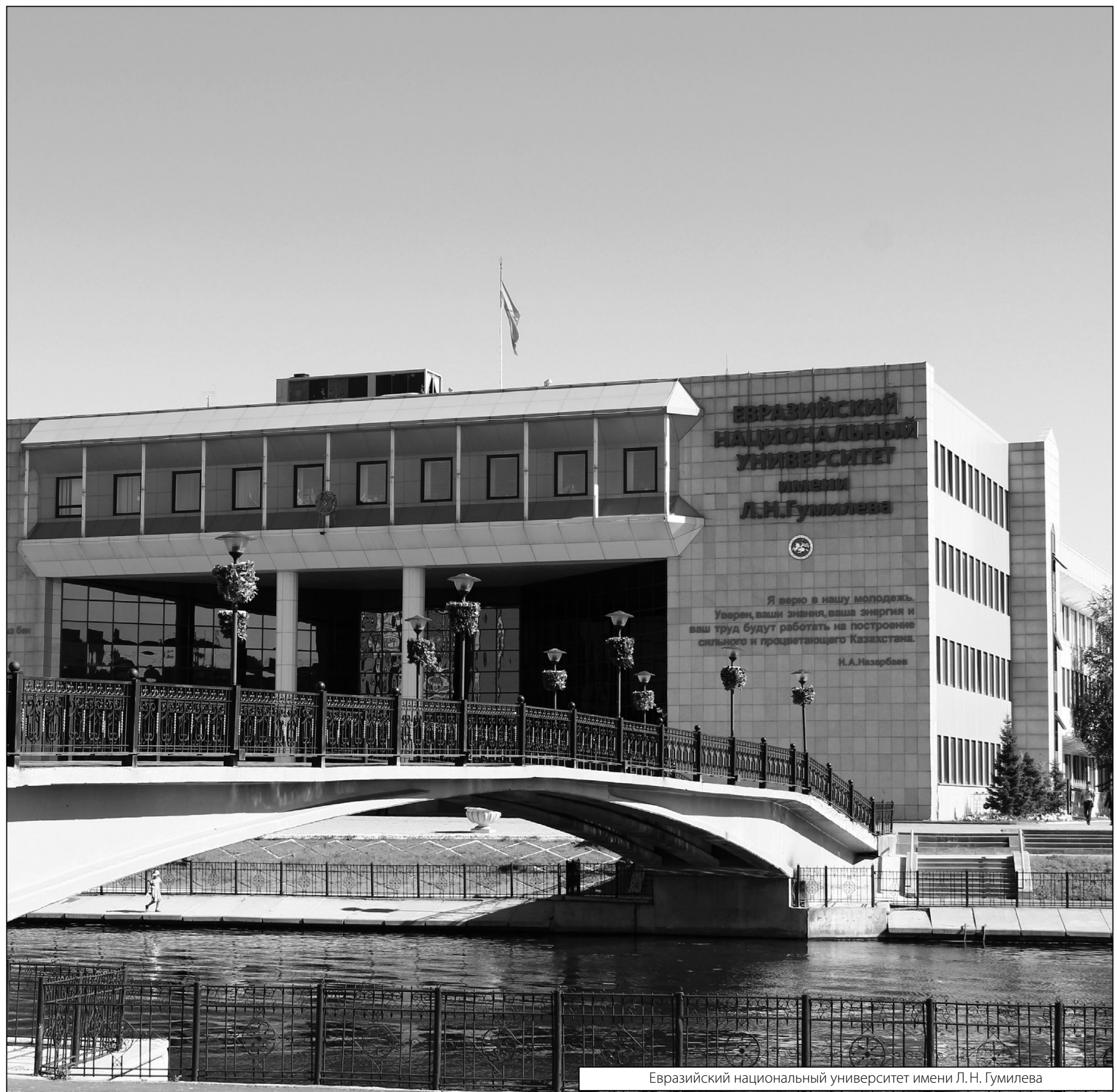

